\title{
A Mini Review on Non-augmentative Surgical Therapy of Peri-Implantitis-What Is Known and What Are the Future Challenges?
}

\author{
Kristina Bertl ${ }^{1,2 *}$ and Andreas Stavropoulos ${ }^{1,3,4}$ \\ ${ }^{1}$ Department of Periodontology, Faculty of Odontology, University of Malmö, Malmö, Sweden, ${ }^{2}$ Division of Oral Surgery, \\ University Clinic of Dentistry, Medical University of Vienna, Vienna, Austria, ${ }^{3}$ Division of Regenerative Dental Medicine and \\ Periodontology, University Clinics of Dental Medicine, University of Geneva, Geneva, Switzerland, ${ }^{4}$ Division of Conservative \\ Dentistry and Periodontology, University Clinic of Dentistry, Medical University of Vienna, Vienna, Austria
}

\section{OPEN ACCESS}

Edited by:

Priscila Casado,

Fluminense Federal University, Brazi

Reviewed by:

Jamil Awad Shibli,

Guarulhos University, Brazil

Danae Anastasia Apatzidou,

Aristotle University of

Thessaloniki, Greece

${ }^{*}$ Correspondence:

Kristina Bert

kristina.bert!@mau.se

Specialty section:

This article was submitted to

Periodontics,

a section of the journal

Frontiers in Dental Medicine

Received: 27 January 2021

Accepted: 25 March 2021

Published: 20 April 2021

Citation:

Bertl K and Stavropoulos A (2021) A

Mini Review on Non-augmentative

Surgical Therapy of

Peri-Implantitis - What Is Known and

What Are the Future Challenges?

Front. Dent. Med. 2:659361.

doi: 10.3389/fdmed.2021.659361
Non-augmentative surgical therapy of peri-implantitis is indicated for cases with primarily horizontal bone loss or wide defects with limited potential for bone regeneration and/or re-osseointegration. This treatment approach includes a variety of different techniques (e.g., open flap debridement, resection of peri-implant mucosa, apically positioned flaps, bone re-contouring, implantoplasty, etc.) and various relevant aspects should be considered during treatment planning. The present mini review provides an overview on what is known for the following components of non-augmentative surgical treatment of peri-implantitis and on potential future research challenges: (1) decontamination of the implant surface, (2) need of implantoplasty, (3) prescription of antibiotics, and (4) extent of resective measures.

Keywords: antibiotics, decontamination, implantoplasty, review, non-augmentative therapy, peri-implantitis

\section{BACKGROUND}

Due to the high number of annually placed dental implants worldwide (i.e., roughly $>12$ million implants are installed per year) in combination with the high prevalence of peri-implant mucositis (ca. 43\%) and peri-implantitis (ca. 22\%), treatment of biological peri-implant complications has become part of everyday praxis $(1,2)$. While the treatment of peri-implant mucositis often leads to a significant reduction in the degree of peri-implant inflammation (i.e., reduced bleeding upon probing) in the majority of cases, the rate of disease recurrence after non-surgical treatment of peri-implantitis is high (3-5), which in turn leads often to the necessity of surgical interventions; these can be either augmentative or non-augmentative in nature. An augmentative or regenerative/reconstructive approach, which includes the application of autogenous bone, bone substitutes, and/or membranes, is primarily limited to those cases with circumferential and intrabony defects with a high potential for bone regeneration; in this context, an augmentative approach seems more relevant for implants with a modified surface, where the potential for reosseointegration is higher compared to a turned (i.e., non-modified) surface $(6,7)$. While for cases with primarily horizontal bone loss or wide defects with reduced potential for bone regeneration a non-augmentative and potentially even resective approach should be chosen (e.g., open flap debridement, resection of peri-implant mucosa, and/or apically positioned flap, with/without bone re-contouring, with/without implantoplasty, etc.); a primarily horizontal bone loss can be found in approximately every fifth implant affected by peri-implant bone loss (8). Although one 
might assume a non-augmentative approach to be technically simpler and more straightforward than an augmentative one, there are many aspects to consider during treatment planning. The present mini review provides an overview on what is known for the following components of non-augmentative surgical treatment of peri-implantitis and on the potential future research challenges: (1) decontamination of the implant surface, (2) need of implantoplasty, (3) prescription of antibiotics, and (4) extent of resection.

\section{Decontamination of the Implant Surface}

The primary aim of implant surface decontamination is complete removal of the bacterial biofilm, in order to provide an optimal environment for undisturbed healing and thereby result in resolution of inflammation. For decontamination, chemical [e.g., hydrogen peroxide, chlorhexidine gluconate, citric acid, etc. in various concentrations, application times and modes (i.e., single vs. repeated application, immersion vs. rubbing)] and mechanical measures (e.g., curettes, ultrasonic devices, airpolishing devices, etc.) or a combination thereof are described in the literature (9). Based on the results of laboratory and preclinical studies it appears that complete biofilm removal from the implant surface is not feasible, and a combination of mechanical and chemical measures should be used (10-12). In a recent clinical trial (13) including 20 patients, each with 4 implants classified as hopeless, the intra-operative efficacy of three different protocols for biofilm removal was compared. The results significantly favored a primarily mechanical (air-polishing device) or mechanical-chemical approach (air-polishing device combined with hydrogen peroxide and chlorhexidine gluconate) compared to a primarily chemical approach (hydrogen peroxide and chlorhexidine gluconate); no significant differences were noticed between the mechanical and the combined mechanicalchemical protocol. Yet, complete biofilm removal was not achieved by any of the tested methods. In this context, laboratory studies simulating a surgical approach with horizontal bone loss and comparing different mechanical measures overall indicate an advantage of air-polishing devices when compared to curettes and ultrasonic devices $(14,15)$. However, a recent literature review on the efficacy of the various decontamination protocols used in clinical trials (9) showed that existing data do not favor a specific protocol irrespective of the type of surgical approach, that is, also not among those studies using a non-augmentative approach (16-21). Further, according to the results of a recent systematic review of the American Academy of Periodontology, neither use of laser technology (i.e., Er:YAG, $\mathrm{CO}_{2}$, diode laser) for surface decontamination during surgical peri-implantitis therapy appears to provide any relevant improvement in clinical parameters, such as pocket depth reduction or clinical attachment level gain (22). In line with these clinical data, a very recent in vitro study (23) failed to show a difference in the efficacy of implant surface decontamination between Er:YAG laser, titanium brushes, and carbon fiber curettes. Implant surface decontamination is thus one of the most challenging topics for future research. For instance, the potential differences in terms of efficacy of different types of air abrasive powders (i.e., sodium bicarbonate powder vs. glycine or erythritol powders) (24), the efficacy of a recently suggested invention based on electrolysis $(25,26)$, or different concentrations and application times and modes of chemical agents (27), in dependence of implant- and thread design (28) and biofilm location (29) are interesting aspects for future trials.

\section{Need of Implantoplasty}

Implantoplasty (i.e., removal of the implant threads and of the micro-structured surface and smoothening of the implant surface by means of rotating instruments during surgical peri-implantitis treatment) might be considered as the only "decontamination measure" actually achieving complete removal of bacterial biofilm (30). Implantoplasty is primarily recommended for implants or aspects of implants with a modified surface (i.e., in contrast to turned, non-modified surfaces), and at those aspects of the implant, where due to the morphology of the bone defect regeneration cannot be expected. Specifically, implantoplasty should be primarily performed at implants with horizontal bone loss and/or buccal/oral dehiscences, but not at those aspects facing intrabony or crater-shaped defects, as these should be subject to augmentative procedures (31). Based on laboratory studies $(32,33)$, depending on the type of burs and polishing instruments used, mean $R_{a}$ (arithmetic mean roughness) and $R_{\mathrm{z}}$ (averaged roughness) values ranging from 0.32 to $0.98 \mu \mathrm{m}$ and from 1.87 to $6.86 \mu \mathrm{m}$, respectively, can approximately be achieved after implantoplasty. Although these values remain higher than industrially polished implant surfaces [e.g., for the polished neck of a specific implant type $R_{a}$ and $R_{z}$ values of 0.1 and $0.81 \mu \mathrm{m}$, respectively, have been reported (32)], a recent proof-of-concept clinical study (34) reported distinctly reduced intra-oral plaque accumulation and biofilm formation on implant surfaces treated with implantoplasty compared to nontreated moderately rough implant surfaces. Hence, the rationale for implantoplasty is to remove any existing biofilm as well as to prevent/reduce postoperative recolonization by creating a surface less conductive to biofilm accumulation $(30,34)$. Although its potential effectiveness has been indicated already about 15 years ago by a randomized controlled clinical trial with a 3-year follow-up $(35,36)$ showing a survival rate of $100 \%$ and no further peri-implant marginal bone loss in the implantoplasty test group, this technique remains controversially discussed. Specifically, the major concerns are a weakening of the implant material leading to a higher rate of implant fracture as well as an overheating of the surrounding bone and a release of titanium particles into the surrounding tissues during the procedure. However, a recent systematic review (37) on mechanical and biological complications after implantoplasty did not confirm these concerns. Specifically, based on the available evidence of laboratory, preclinical, and clinical trials it was concluded, that i) implantoplasty does not result in temperature increase provided proper cooling is used; ii) titanium particle deposition in the surrounding tissues is possible and implantoplasty leads on the bench to reduced implant strength in standard/regular diameter implants, but iii) no clinical study has reported any remarkable complication due to implantoplasty (i.e., among almost 300 implants treated with implantoplasty no implant fracture was reported after a follow-up of 3 to 126 months and only a 
single case of mucosal discoloration was described). Further, a more recently published laboratory study (38), including regular and narrow diameter implants confirmed that although implantoplasty reduces (on the bench) the implant failure strength, this will hardly affect the majority of the cases in the clinic, since implants still showed high maximum implant failure strength [i.e., forces in this specific study remained for most implant types $>440 \mathrm{~N}$, while forces occurring in the natural dentition during regular mastication range between 100 and $300 \mathrm{~N}$ (39)]. This study indicated that only single narrow titanium tissue level implants may have an increased risk of mechanical complications. Altogether, the decision to which
A

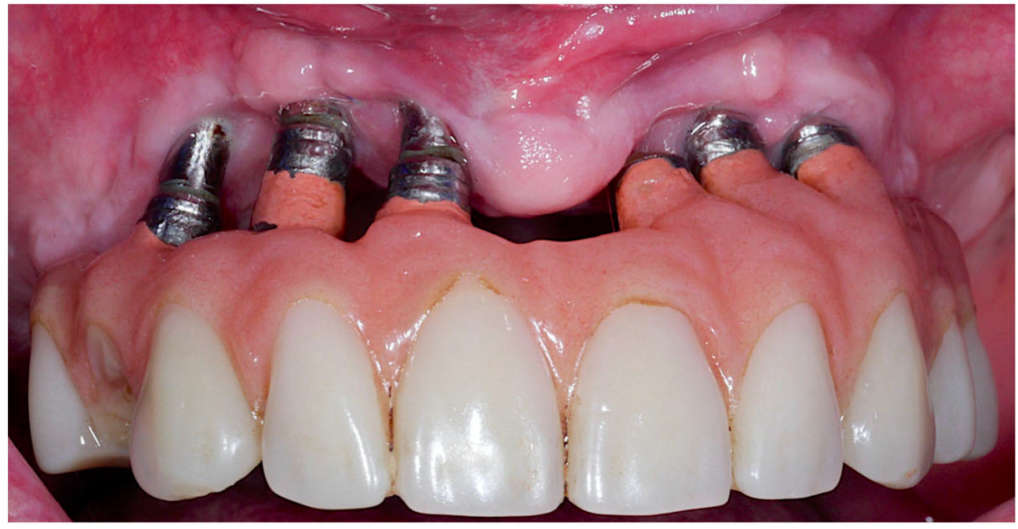

C

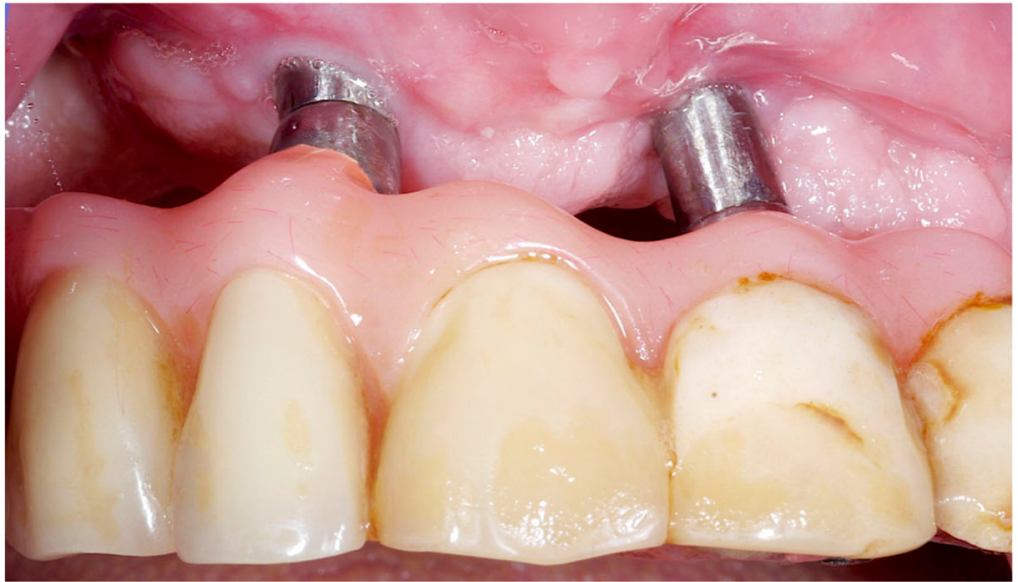

E

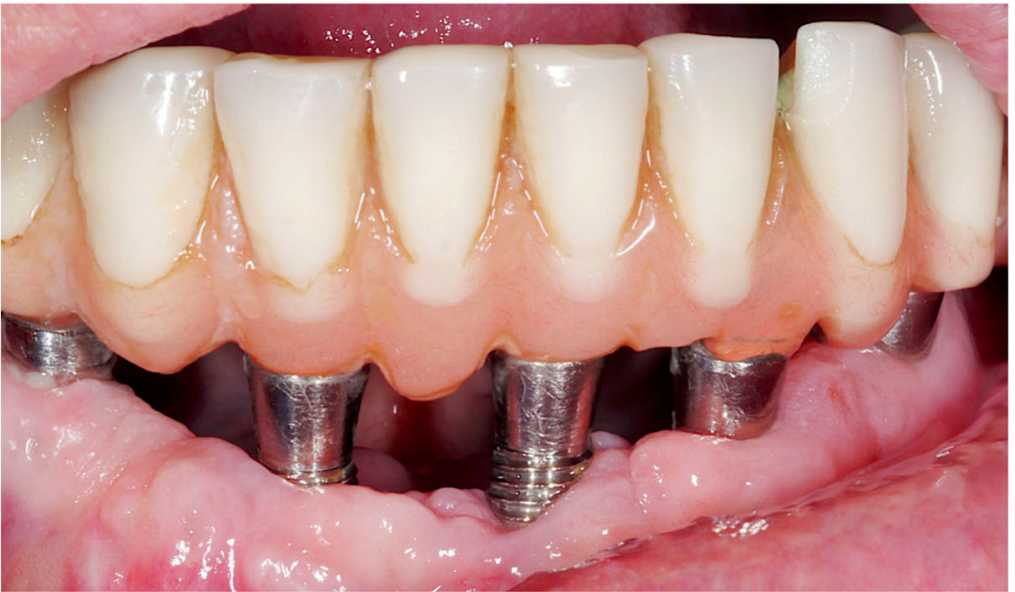

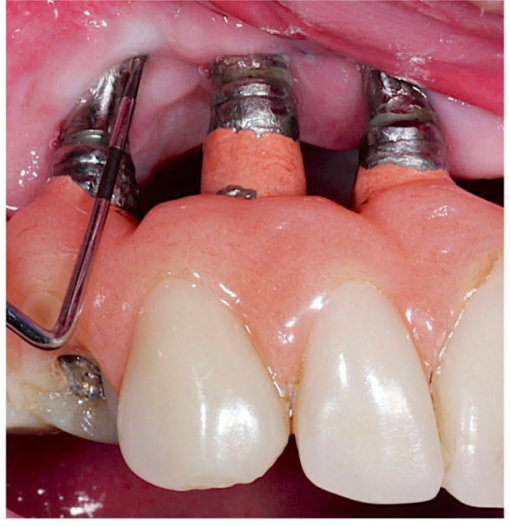

B
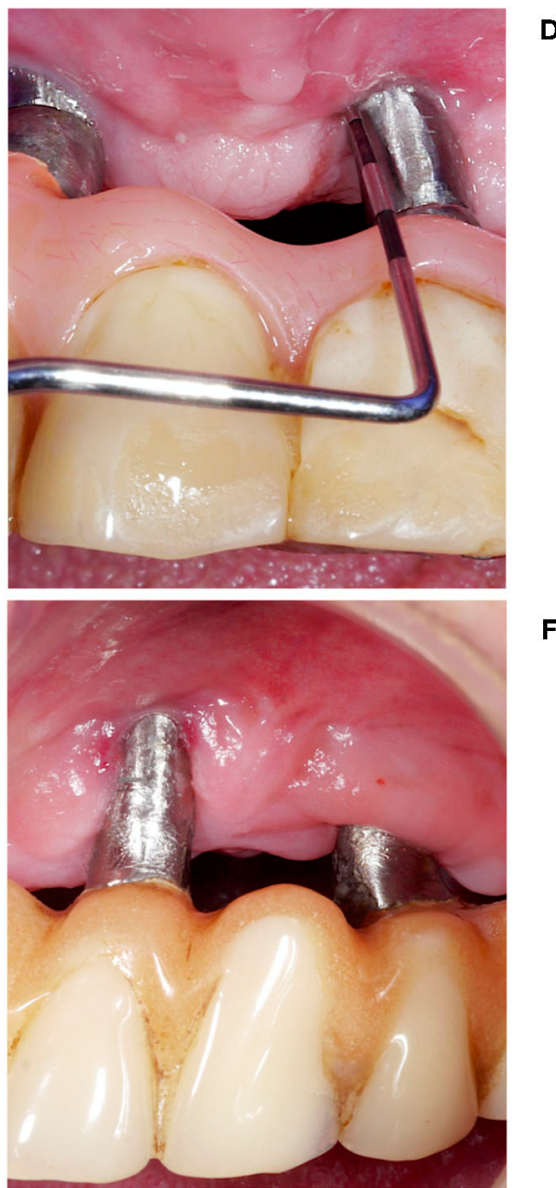

FIGURE 1 | Four patient cases (A, C, E, F), which have been treated surgically with non-augmentative, resective approaches. The resective treatment measures included in all cases a paramarginal incision, an apically positioned flap, and bone re-contouring where indicated. This provided postoperatively shallow pockets without signs of persistent inflammation (B, D), but was associated with an aesthetically compromised outcome (A, C, E, F). However, in these specific cases a low smile line and/or low aesthetic concerns of the patients allowed to choose such an approach. 
extent implantoplasty is meaningful in a particular case depends on various parameters, such as implant dimension (diameter, length) and material (titanium vs. titanium-zirconium alloy), prosthetic restoration (single- vs. multi-unit), and/or aesthetic demands of the patient. However, based on the available evidence straightforward guidelines and/or clear contraindications when and when not to perform implantoplasty cannot be provided; nevertheless, the fact of an actual lack of reports on implant fracture after implantoplasty indicates that this "worst case scenario" probably occurs rather seldom. Considering for the clinical outcome more recently published clinical trials, the results remain partly controversial and cannot confirm or reject the performance of implantoplasty. Specifically, in 2 case series with $\geq 2$ years of follow-up after a combined resective-implantoplasty approach, approximately $90 \%$ of the implants presented with disease resolution and/or stable bone levels $(40,41)$, while a retrospective analysis failed to show a significant advantage of performing implantoplasty in addition to a resective approach (42). However, in the latter, more implants in the implantoplasty group presented with a bone loss $\geq 25 \%$ compared to the control group not receiving implantoplasty (i.e., 66.6 vs. $39.5 \%$, respectively). Another recently published randomized controlled clinical trial (43) compared the efficacy of implantoplasty with an air-polishing device while performing an open flap debridement without bone re-contouring. Within a limited follow-up period of 6 months, which is too short to show differences in disease recurrence, no significant differences were observed for one of the two treatment approaches. Further, a prospective longitudinal study (44) with 3 years followup compared chemical decontamination (with citric acid) to chemical decontamination with a subepithelial connective tissue graft and to chemical decontamination with implantoplasty. Survival rate $(100 \%)$ and rate of disease resolution $(62.5 \%)$ was highest in the implantoplasty group but lacked statistically significance compared to the other two groups. However, the baseline peri-implant marginal bone levels presented also herein clinically relevant differences ranging from 4.3 to $5.5 \mathrm{~mm}$ with the implantoplasty group presenting the highest values. Finally, taking the results of recent studies on non-augmentative, resective approaches-not performing implantoplasty-into account, indicate the potential relevance of the implant surface characteristic for treatment decision. Specifically, in these studies $(17,45)$ the outcome was superior at implants with a turned surface compared to implants with a modified surface. Hence, one might conclude that implants with a modified surface need a more efficient decontamination protocol, and implantoplasty could be a way to achieve this. Altogether, although the results of the first randomized controlled clinical trial $(35,36)$ clearly favor to perform implantoplasty, it is relevant to perform welldesigned, prospective, randomized controlled clinical trials with sufficient power and follow-up period to either confirm or reject implantoplasty in combination within a non-augmentative treatment approach of peri-implantitis.

\section{Prescription of Antibiotics}

The results of two recent surveys indicated, that a relatively high percentage of practitioners appears to prescribe systemic antibiotics in combination with peri-implantitis treatment; that is, 24 to $44 \%$ and 31 to $34 \%$ indicated to use systemic antibiotics "always" and "often," respectively $(46,47)$. The rationale to use antibiotics in non-augmentative procedures would be primarily to improve implant surface decontamination, as disrupted but residual biofilm components are supposedly more susceptible against antibiotics, while in augmentative procedures systemic antibiotics are also intended to provide post-operative infection control. However, the effect size of systemic antibiotics as adjunct to surgical treatment in non-augmentative cases is still discussed controversially and the number of available comparative studies is limited. Specifically, short-term reports (i.e., up to 12 months follow-up) report either no statistically significant effect prescribing azithromycin $(250 \mathrm{mg}$ twice at the day of surgery followed by $250 \mathrm{mg}$ once per day for 4 additional days) (48) or an effect depending on the implant surface, with systemic intake of antibiotics (i.e., amoxicillin $750 \mathrm{mg}$ twice daily for 10 days starting 3 days prior to surgery) providing additional benefit only in cases with implants with modified surfaces (16); any potential effect, however, appears not sustainable over a longer period of time, that is, over a follow-up period of 3 years (17). For comparison, a long-term prospective clinical study (49) using open flap debridement in combination with systemic intake of antibiotics (i.e., amoxicillin $500 \mathrm{mg}$ and metronidazole $400 \mathrm{mg}$ each 3-times daily for 1 week) reported from 1 to 5 years a reduction in the success rate from 79 to $63 \%$ and 81 to $53 \%$ on the patient- and implant-level, respectively. As an alternative to systemic antibiotics, a recently published randomized controlled clinical trial reported favorable results when combining open flap debridement with repeated local application of minocycline (50). However, although the local application would provide certain advantages, such as a reduced risk for antibiotic resistances, the report is limited to a 6-month follow-up and therefore longterm reports are warranted. Altogether, future well-designed randomized controlled clinical trials with sufficient power should confirm or reject the prescription of systemic antibiotics vs. application of local antibiotics within a non-augmentative treatment approach. Specifically, in terms of antibiotic intake, the long-term benefit as well as the type and duration of antibiotic intake depending on implant- (e.g., turned or modified implant surface) and patient-characteristics (e.g., smoking status, systemic diseases) are highly interesting.

\section{Extent of Resective Measures}

Non-augmentative surgical treatment of peri-implantitis includes a variety of different surgical techniques and combinations thereof, that is, open flap debridement, resection of peri-implant mucosa, apically positioned flap, bone recontouring, implantoplasty, etc. While the advantages and disadvantages of implantoplasty have been outlined above, flap manipulation and bone re-contouring might positively affect the extent of pocket elimination, but often negatively the aesthetic outcome (41). Specifically, a resective approach including paramarginal incisions, apically positioning of the flap, bone re-contouring, and/or implantoplasty can provide postoperative shallow pockets, but is often associated with an aesthetically compromised outcome (Figure 1). Hence, 
treatment planning for non-augmentative cases and the choice between a simple open flap debridement and a more resective approach should take implant position, smile line, and aesthetic concerns of the patient into account. Currently, relatively little is known on the need of extensive resective measures to maintain long-term stability. Based on the results of few available long-term studies applying either open flap debridement only (49) or a resective approach including bone re-contouring where indicated $(17,45)$, it seems that both treatment approaches are effective in controlling peri-implantitis in the majority of the patients and implants; however, a direct comparison of these techniques could be one of the future challenges.

\section{CONCLUSIONS}

Non-augmentative treatment approaches are often performed on implants with substantial, mostly horizontal bone loss. The decision whether such an implant should be kept and treated or actually explanted (and potentially replaced) is often a quite individual decision and depends on several implant(e.g., implant dimension and material), prosthetic- (e.g., singlevs. multi-unit, need of this specific implant to maintain the prosthetic restoration), and patient-related parameters [e.g., aesthetic demands, economic possibilities, willingness to and/or physical capability for (extensive) re-treatment]. However, if performed the outcome should provide easy access to oral

\section{REFERENCES}

1. Derks J, Tomasi C. Peri-implant health and disease. A systematic review of current epidemiology. J Clin Periodontol. (2015) 42(Suppl 16):S158-71. doi: $10.1111 /$ jcpe. 12334

2. Klinge B, Klinge A, Bertl K, Stavropoulos A. Peri-implant diseases. Eur J Oral Sci. (2018) 126(Suppl 1):88-94. doi: 10.1111/eos.12529

3. Figuero E, Graziani F, Sanz I, Herrera D, Sanz M. Management of periimplant mucositis and peri-implantitis. Periodontol 2000. (2014) 66:255-73. doi: $10.1111 /$ prd.12049

4. Renvert S, Polyzois I. Treatment of pathologic peri-implant pockets. Periodontol 2000. (2018) 76:180-90. doi: 10.1111/prd.12149

5. Suárez-López Del Amo F, Yu SH, Wang HL. Non-surgical therapy for periimplant diseases: a systematic review. J Oral Maxillofac Res. (2016) 7:e13. doi: 10.5037/jomr.2016.7313

6. Persson LG, Berglundh T, Lindhe J, Sennerby L. Re-osseointegration after treatment of peri-implantitis at different implant surfaces. An experimental study in the dog. Clin Oral Implants Res. (2001) 12:595-603.

7. Renvert S, Polyzois I, Maguire R. Re-osseointegration on previously contaminated surfaces: a systematic review. Clin Oral Implants Res. (2009) 20(Suppl 4):216-27. doi: 10.1111/j.1600-0501.2009.01786.x

8. Wehner C, Bertl K, Durstberger G, Arnhart C, Rausch-Fan X, Stavropoulos A. Characteristics and frequency distribution of bone defect configurations in peri-implantitis lesions-A series of 193 cases. Clin Implant Dent Relat Res. (2020). doi: 10.1111/cid.12961. [Epub ahead of print].

9. Koo KT, Khoury F, Keeve PL, Schwarz F, Ramanauskaite A, Sculean A, et al. Implant surface decontamination by surgical treatment of periimplantitis: a literature review. Implant Dent. (2019) 28:173-6. doi: 10.1097/ID.0000000000000840

10. Louropoulou A, Slot DE, Van der Weijden F. The effects of mechanical instruments on contaminated titanium dental implant surfaces: a systematic review. Clin Oral Implants Res. (2014) 25:1149-60. doi: 10.1111/clr.12224 hygiene and maintenance measures and most of all achieve disease resolution to avoid persistent local inflammation with the risk for a systemic impact as well as continuous periimplant bone loss, which might further impede/complicate future replacement of the implant. Altogether, non-augmentative treatment approaches will remain an important part of the array of surgical treatment modalities of peri-implantitis, especially in non-aesthetic cases. Considering the available evidence on the aspects discussed above (i.e., decontamination of the implant surface, need of implantoplasty, prescription of antibiotics, and extent of resective measures), it is clear, that there are many future research challenges and that the standard of surgical interventions to control periodontitis has not been reached for peri-implantitis. Beside these aspects and independent of the treatment modality (i.e., augmentative or non-augmentative), the usefulness of a recently introduced risk profile (51) as well as the frequency of supportive treatment and the potential of local adjuncts to prevent re-infection should receive attention in future studies.

\section{AUTHOR CONTRIBUTIONS}

$\mathrm{KB}$ and AS are responsible for concept/design, data collection, drafting of the article, critical revision of the article, and final approval of the article. All authors contributed to the article and approved the submitted version.

11. Ntrouka VI, Slot DE, Louropoulou A, Van der Weijden F. The effect of chemotherapeutic agents on contaminated titanium surfaces: a systematic review. Clin Oral Implants Res. (2011) 22:681-90. doi: 10.1111/j.1600-0501.2010.02037.x

12. Subramani K, Wismeijer D. Decontamination of titanium implant surface and re-osseointegration to treat peri-implantitis: a literature review. Int J Oral Maxillofac Implants. (2012) 27:1043-54.

13. Pranno N, Cristalli MP, Mengoni F, Sauzullo I, Annibali S, Polimeni A, et al. Comparison of the effects of air-powder abrasion, chemical decontamination, or their combination in open-flap surface decontamination of implants failed for peri-implantitis: an ex vivo study. Clin Oral Investig. (2020). doi: 10.1007/s00784-020-03578-w. [Epub ahead of print].

14. Keim D, Nickles K, Dannewitz B, Ratka C, Eickholz P, Petsos H. In vitro efficacy of three different implant surface decontamination methods in three different defect configurations. Clin Oral Implants Res. (2019) 30:550-8. doi: $10.1111 /$ clr.13441

15. Sahrmann P, Ronay V, Hofer D, Attin T, Jung RE, Schmidlin PR. In vitro cleaning potential of three different implant debridement methods. Clin Oral Implants Res. (2015) 26:314-9. doi: 10.1111/clr.12322

16. Carcuac O, Derks J, Charalampakis G, Abrahamsson I, Wennström J, Berglundh T. Adjunctive systemic and local antimicrobial therapy in the surgical treatment of peri-implantitis: a randomized controlled clinical trial. J Dent Res. (2016) 95:50-7. doi: 10.1177/0022034515601961

17. Carcuac O, Derks J, Abrahamsson I, Wennström JL, Petzold M, Berglundh T. Surgical treatment of peri-implantitis: 3-year results from a randomized controlled clinical trial. J Clin Periodontol. (2017) 44:1294-303. doi: $10.1111 /$ jcpe. 12813

18. de Waal YC, Raghoebar GM, Huddleston Slater JJ, Meijer HJ, Winkel EG, van Winkelhoff AJ. Implant decontamination during surgical peri-implantitis treatment: a randomized, double-blind, placebocontrolled trial. J Clin Periodontol. (2013) 40:186-95. doi: 10.1111/jcpe. 12034 
19. de Waal YC, Raghoebar GM, Meijer HJ, Winkel EG, van Winkelhoff AJ. Implant decontamination with $2 \%$ chlorhexidine during surgical periimplantitis treatment: a randomized, double-blind, controlled trial. Clin Oral Implants Res. (2015) 26:1015-23. doi: 10.1111/clr.12419

20. Hentenaar DFM, De Waal YCM, Strooker H, Meijer HJA, Van Winkelhoff AJ, Raghoebar GM. Implant decontamination with phosphoric acid during surgical peri-implantitis treatment: a RCT. Int J Implant Dent. (2017) 3:33. doi: 10.1186/s40729-017-0091-5

21. Toma S, Lasserre JF, Taïeb J, Brecx MC. Evaluation of an air-abrasive device with amino acid glycine-powder during surgical treatment of peri-implantitis. Quintessence Int. (2014) 45:209-19. doi: 10.3290/j.qi.a31205

22. Lin GH, Suárez López Del Amo F, Wang HL. Laser therapy for treatment of peri-implant mucositis and peri-implantitis: an American Academy of Periodontology best evidence review. J Periodontol. (2018) 89:766-82. doi: 10.1902/jop.2017.160483

23. AlMoharib HS, Steffensen B, Zoukhri D, Finkelman M, Gyurko R. Efficacy of an Er:YAG laser in the decontamination of dental implant surfaces: an in vitro study. J Periodontol. (2021). doi: 10.1002/JPER.20-0765. [Epub ahead of print].

24. Matsubara VH, Leong BW, Leong MJL, Lawrence Z, Becker T, Quaranta A. Cleaning potential of different air abrasive powders and their impact on implant surface roughness. Clin Implant Dent Relat Res. (2020) 22:96-104. doi: $10.1111 /$ cid.12875

25. Schlee M, Rathe F, Brodbeck U, Ratka C, Weigl P, Zipprich H. Treatment of peri-implantitis-electrolytic cleaning versus mechanical and electrolytic cleaning-a randomized controlled clinical trial-six-month results. J Clin Med. (2019) 8:1909. doi: $10.3390 / \mathrm{jcm} 8111909$

26. Schlee M, Naili L, Rathe F, Brodbeck U, Zipprich H. Is complete reosseointegration of an infected dental implant possible? histologic results of a dog study: a short communication. J Clin Med. (2020) 9:235. doi: $10.3390 / \mathrm{jcm} 9010235$

27. Cordeiro JM, Pires JM, Souza JGS, Lima CV, Bertolini MM, Rangel EC, et al. Optimizing citric acid protocol to control implant-related infections: an in vitro and in situ study. J Periodontal Res. (2021). doi: 10.1111/jre.12855. [Epub ahead of print].

28. Sanz-Martín I, Paeng K, Park H, Cha JK, Jung UW, Sanz M. Significance of implant design on the efficacy of different peri-implantitis decontamination protocols. Clin Oral Investig. (2020). doi: 10.1007/s00784-020-03681-y. [Epub ahead of print].

29. Steiger-Ronay V, Merlini A, Wiedemeier DB, Schmidlin PR, Attin T, Sahrmann P. Location of unaccessible implant surface areas during debridement in simulated peri-implantitis therapy. BMC Oral Health. (2017) 17:137. doi: 10.1186/s12903-017-0428-8

30. El Chaar E, Almogahwi M, Abdalkader K, Alshehri A, Cruz S, Ricci J. Decontamination of the infected implant surface: a scanning electron microscope study. Int J Periodontics Restorative Dent. (2020) 40:395-401. doi: $10.11607 /$ prd.4568

31. Solderer A, Schmidlin PR. Regenerative surgical therapy of periimplantitis: an umbrella review of answered/unanswered questions and future perspectives. Front Dent Med. (2020) 1:614240. doi: 10.3389/fdmed.2020.614240

32. Ramel CF, Lüssi A, Özcan M, Jung RE, Hämmerle CH, Thoma DS. Surface roughness of dental implants and treatment time using six different implantoplasty procedures. Clin Oral Implants Res. (2016) 27:776-81. doi: $10.1111 /$ clr.12682

33. Sahrmann P, Luso S, Mueller C, Ender A, Attin T, Stawarczyk B, et al. Titanium implant characteristics after implantoplasty: an in vitro study on two different kinds of instrumentation. Int J Oral Maxillofac Implants. (2019) 34:1299-305. doi: $10.11607 /$ jomi.7410

34. Azzola F, Ionescu AC, Ottobelli M, Cavalli N, Brambilla E, Corbella S, et al. Biofilm formation on dental implant surface treated by implantoplasty: an in situ study. Dent J. (2020) 8:40. doi: 10.3390/dj8020040

35. Romeo E, Ghisolfi M, Murgolo N, Chiapasco M, Lops D, Vogel G. Therapy of peri-implantitis with resective surgery. A 3-year clinical trial on rough screwshaped oral implants. Part I: clinical outcome. Clin Oral Implants Res. (2005) 16:9-18. doi: 10.1111/j.1600-0501.2004.01084.x
36. Romeo E, Lops D, Chiapasco M, Ghisolfi M, Vogel G. Therapy of periimplantitis with resective surgery. A 3-year clinical trial on rough screwshaped oral implants. Part II: radiographic outcome. Clin Oral Implants Res. (2007) 18:179-87. doi: 10.1111/j.1600-0501.2006.01318.x

37. Stavropoulos A, Bertl K, Eren S, Gotfredsen K. Mechanical and biological complications after implantoplasty-a systematic review. Clin Oral Implants Res. (2019) 30:833-48. doi: 10.1111/clr.13499

38. Bertl K, Isidor F, von Steyern PV, Stavropoulos A. Does implantoplasty affect the failure strength of narrow and regular diameter implants? A laboratory study. Clin Oral Investig. (2020) 25:2203-11. doi: 10.1007/s00784-020-03534-8

39. Vallittu PK, Könönen M. Biomechanical aspects and material properties. In: Nilner K, Karlsson S, Dahl BL, editors. A Textbook of Fixed Prosthodontics: The Scandinavian Approach. Stockholm: Gothia Fortbildning (2013). p. 116-30.

40. Bianchini MA, Galarraga-Vinueza ME, Apaza-Bedoya K, De Souza JM, Magini R, Schwarz F. Two to six-year disease resolution and marginal bone stability rates of a modified resective-implantoplasty therapy in 32 peri-implantitis cases. Clin Implant Dent Relat Res. (2019) 21:758-65. doi: $10.1111 /$ cid.12773

41. Englezos E, Cosyn J, Koole S, Jacquet W, De Bruyn H. Resective treatment of peri-implantitis: clinical and radiographic outcomes after 2 years. Int $J$ Periodontics Restorative Dent. (2018) 38:729-35. doi: 10.11607/prd.3386

42. Ravidà A, Siqueira R, Saleh I, Saleh MHA, Giannobile A, Wang HL. Lack of clinical benefit of implantoplasty to improve implant survival rate. J Dent Res. (2020) 99:1348-55. doi: 10.1177/0022034520944158

43. Lasserre JF, Brecx MC, Toma S. Implantoplasty versus glycine air abrasion for the surgical treatment of peri-implantitis: a randomized clinical trial. Int J Oral Maxillofac Implants. (2020) 35:197-206. doi: 10.11607/jomi.6677

44. Dalago HR, Perrotti V, Torres de Freitas SF, Ferreira CF, Piattelli A, Iaculli F, et al. Prospective longitudinal comparison study of surgical therapies for peri-implantitis: 3-year follow-up. Aust Dent J. (2019) 64:237-45. doi: 10.1111 /adj. 12693

45. Berglundh T, Wennström JL, Lindhe J. Long-term outcome of surgical treatment of peri-implantitis. A 2-11-year retrospective study. Clin Oral Implants Res. (2018) 29:404-10. doi: 10.1111/clr.13138

46. Khan A, Sharma D. Management of peri-implant diseases: a survey of Australian periodontists. Dent J. (2020) 8:100. doi: 10.3390/dj80 30100

47. Papathanasiou E, Finkelman M, Hanley J, Parashis AO. Prevalence, etiology and treatment of peri-implant mucositis and peri-implantitis: a survey of us periodontists. J Periodontol. (2016) 87:493-501. doi: 10.1902/jop.2015. 150476

48. Hallström H, Persson GR, Lindgren S, Renvert S. Open flap debridement of peri-implantitis with or without adjunctive systemic antibiotics: a randomized clinical trial. J Clin Periodontol. (2017) 44:1285-93. doi: 10.1111/jcpe. 12805

49. Heitz-Mayfield LJA, Salvi GE, Mombelli A, Loup PJ, Heitz F, Kruger E, et al. Supportive peri-implant therapy following anti-infective surgical periimplantitis treatment: 5-year survival and success. Clin Oral Implants Res. (2018) 29:1-6. doi: 10.1111/clr.12910

50. Cha JK, Lee JS, Kim CS. Surgical therapy of peri-implantitis with local minocycline: a 6-month randomized controlled clinical trial. J Dent Res. (2019) 98:288-95. doi: 10.1177/0022034518818479

51. Heitz-Mayfield LJA, Heitz F, Lang NP. Implant disease risk assessment IDRAa tool for preventing peri-implant disease. Clin Oral Implants Res. (2020) 31:397-403. doi: 10.1111/clr.13585

Conflict of Interest: The authors declare that the research was conducted in the absence of any commercial or financial relationships that could be construed as a potential conflict of interest.

Copyright $\odot 2021$ Bertl and Stavropoulos. This is an open-access article distributed under the terms of the Creative Commons Attribution License (CC BY). The use, distribution or reproduction in other forums is permitted, provided the original author(s) and the copyright owner(s) are credited and that the original publication in this journal is cited, in accordance with accepted academic practice. No use, distribution or reproduction is permitted which does not comply with these terms. 